\title{
July 2017 Pulmonary Case of the Month
}

Robert W. Viggiano, MD

\author{
Department of Pulmonary Medicine \\ Mayo Clinic Arizona \\ Scottsdale, AZ USA
}

\section{History of Present IIIness}

The patient is a 19-year-old woman who went to a local Emergency Room 12/23/15 for chest pain she described as pleurisy. She was told she had pneumonia and a chest $\mathrm{x}$ ray was reported to show a lingular infiltrate (Figure 1).

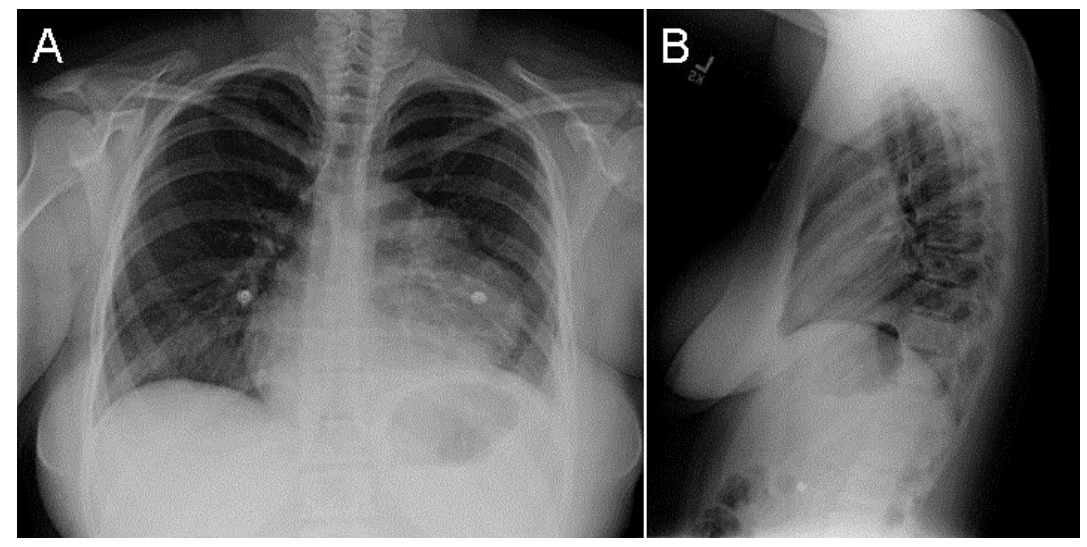

Figure 1. PA (A) and lateral (B) chest radiograph taken 12/23/15.

She was treated with antibiotics and improved. She was well until 9/2/16 when she again returned to the emergency room complaining of hemoptysis. A chest $\mathrm{x}$-ray was reported as showing a lingular infiltrate (Figure 2).

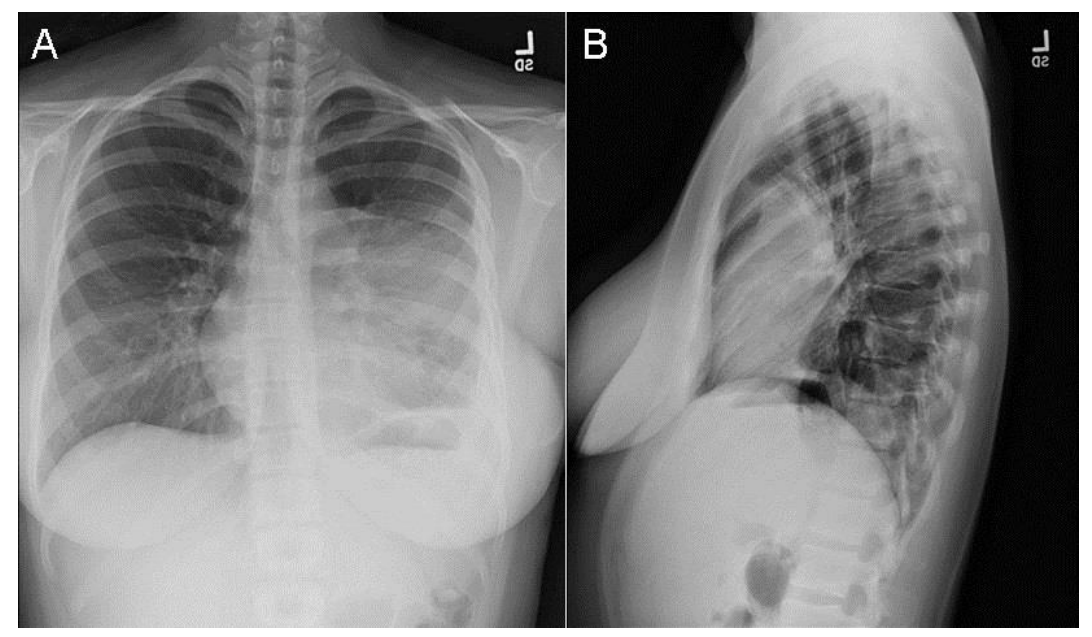

Figure 2. PA (A) and lateral (B) chest radiograph taken 9/2/16. 
She was treated with azithromycin but her cough persisted sometimes with a small amount of blood in her sputum. She was referred because of her persistent symptoms and her abnormal chest x-ray.

\section{Past Medical History, Social History and Family History}

- She is now taking fluoxetine daily.

- She has a history of pediatric autoimmune neuropsychiatric disorder associated with Group A Streptococcus and was treated with antibiotics for 4-5 years.

- Nonsmoker.

\section{Physical Examination}

Her physical examination was unremarkable.

Which of the following are true?

1. Her chest radiographs are consistent with pneumonia

2. Lung cancer is an unlikely consideration in a 19-year-old

3. The chest $x$-ray findings represent a well-known complication of pediatric autoimmune neuropsychiatric disorder

4. 1 and 3

5. All of the above 


\section{Correct!}

\section{Lung cancer is an unlikely consideration in a 19-year-old}

There is an enlarging density in the left chest which is obscuring the left heart border. Lung cancer would be quite unlikely in a 19-year-old. The lack of clearing on the chest $x$-ray is not consistent with community-acquired pneumonia. Pediatric autoimmune neuropsychiatric disorders associated with streptococcal infections is the presence of obsessive compulsive and/or a tic disorder with prepubertal symptom onset in association with a streptococcal infection (1). Long term sequelae are unknown.

Which of the following are true?

1. A thoracic CT scan should be done next

2. Another course of antibiotics is appropriate

3. The density is located in the anterior chest

4. 1 and 3

5. All of the above 


\section{Correct!}

\section{1 and 3}

It seems unlikely another course of antibiotics is likely to cure a clinical course and chest x-ray not consistent with pneumonia. The heart separates the anterior from the middle chest and a lesion silhouetting the heart would be located in the anterior or middle chest. The next step in her evaluation would be to perform a thoracic CT scan (Figure 3).
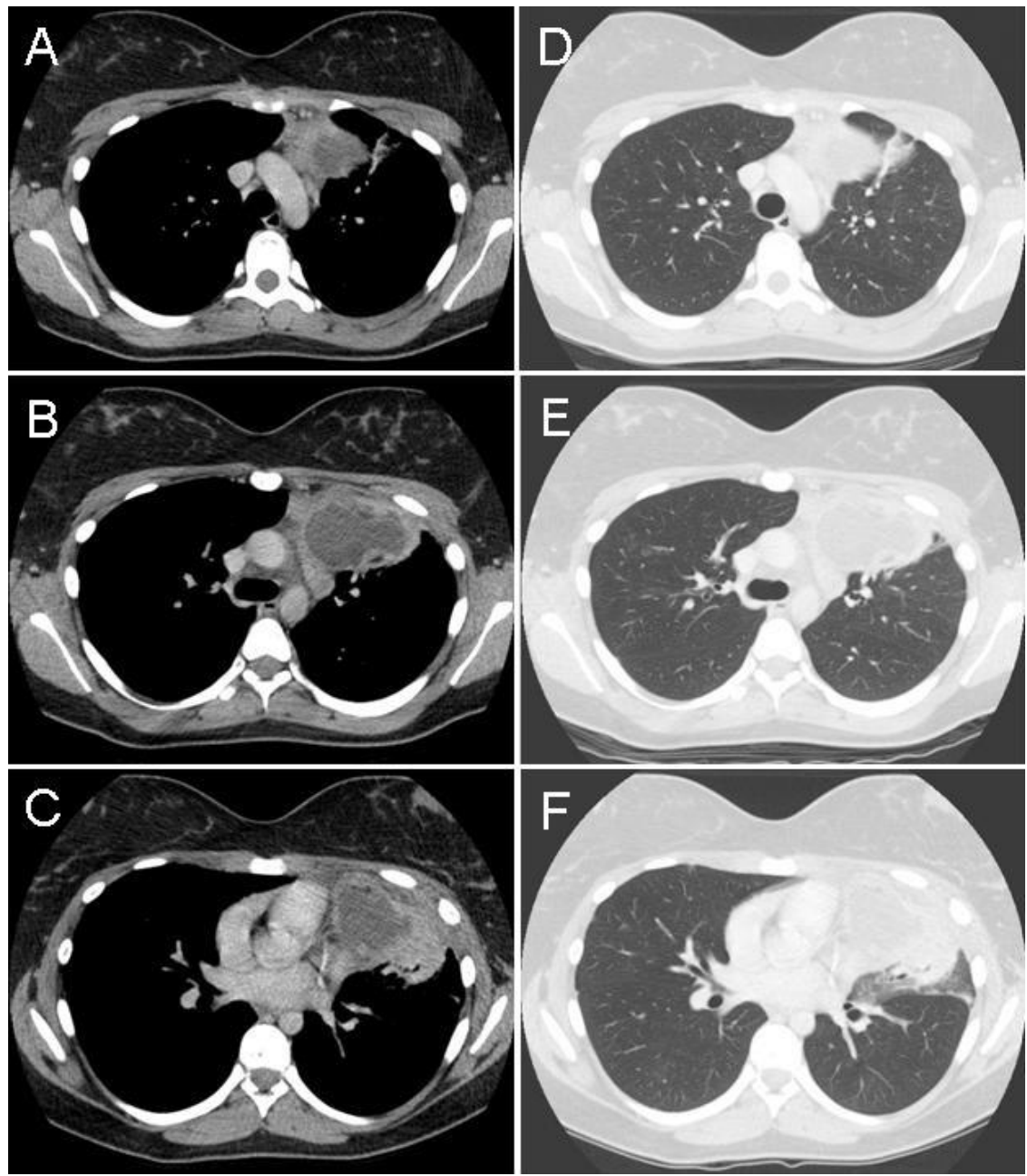

Figure 3. Representative views of the thoracic CT scan in soft tissue windows (A-C) and corresponding lung windows (D-F).

Which of the following are $\underline{\text { true? }}$ ?

1. There is a pericardial cyst

2. There is an anterior mediastinal mass

3. There is an esophageal mass

4. 1 and 3

5. All of the above 


\section{Correct! \\ 2. There is an anterior mediastinal mass}

The mass is in the anterior mediastinum extending to the left side of the thorax anterior to the lung (2). Although it does abut the pericardium, the mass appears outside the pericardium. An esophageal mass would be expected to be more posterior.

The mediastinum is divided by the heart into anterior, middle and posterior compartments (Figure 4).

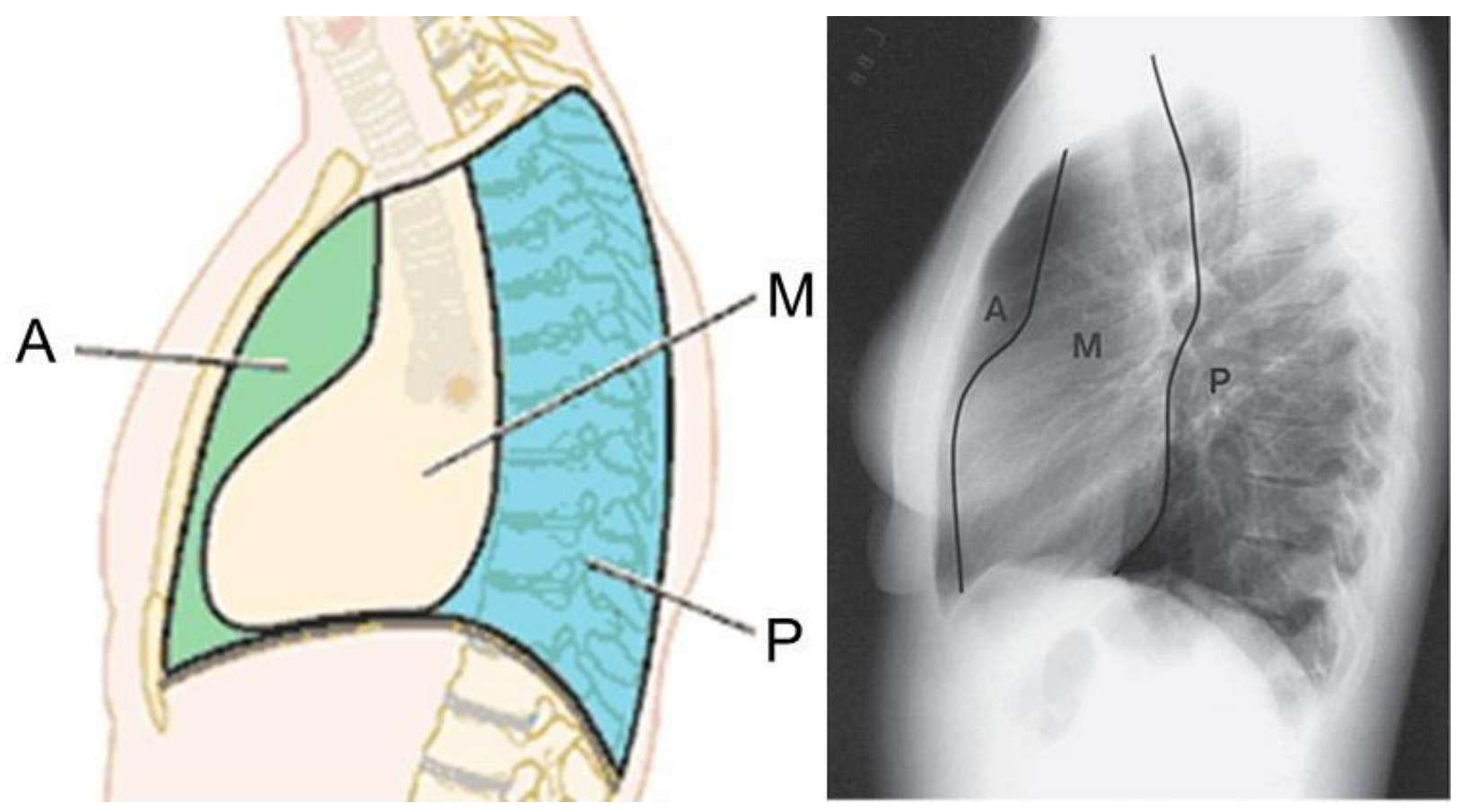

Figure 4. Left: Illustration on the left of the anterior $(\mathrm{A})$ compartment in green, the middle $(\mathrm{M})$ in tan and the posterior $(\mathrm{P})$ in blue. Right: normal lateral chest radiograph on the left shows the corresponding compartments.

Which of the following is the most likely diagnosis?

1. Lymphoma

2. Teratoma

3. Thoracic aortic aneurysm

4. Thymoma

5. Thyroid tumor 


\section{Correct!}

\section{Teratoma}

All are causes of an anterior mediastinal mass. However, given the heterogenous radiographic appearance, the slow progression and the patient's age, the most likely diagnosis is a teratoma (4).

Teratomas are the most common germ cell tumors, occur equally in males and females and usually present at 20 to 40 years of age. About $80 \%$ are benign. A third have symptoms due to compression and obstruction of surrounding organs including chest pain, cough, or dyspnea. They may cause bronchial obstruction and post-obstructive pneumonia. Expectoration of hair (trichoptysis) or sebaceous debris is pathognomonic of benign teratoma, and results from erosion into an adjacent bronchus.

Chest $x$-rays may demonstrate an anterior mediastinal mass, with calcification seen in $26 \%$ of benign teratomas. Teeth or bone can be seen on plain chest $x$-rays and thoracic CT scans or MRI can characterize densities within the lesion suggestive of fat, sebaceous material, or cystic elements.

Our patient underwent resection of the mass which was a typical benign teratoma (Figure 5).

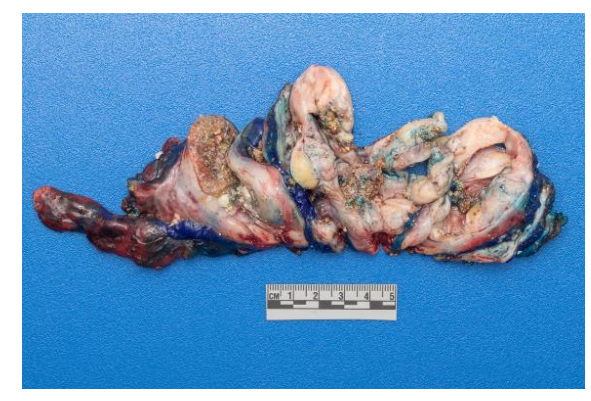

Figure 5. Gross pathology of the resected anterior mediastinal mass showing a heterogenous mass sebaceous and fatty areas.

She made an uneventful recovery and is doing well on follow-up.

\section{References}

1. Swedo SE, Leonard HL, Garvey M, Mittleman B, Allen AJ, Perlmutter S, Lougee L, Dow S, Zamkoff J, Dubbert BK. Pediatric autoimmune neuropsychiatric disorders associated with streptococcal infections: clinical description of the first 50 cases. Am J Psychiatry. 1998 Feb;155(2):264-71. [CrossRef]

2. Berry MF. Approach to the adult patient with a mediastinal mass. UpToDate. September 28, 2016. Available at: https://www.uptodate.com/contents/approach-tothe-adult-patient-with-a-mediastinal-mass\#H16465655 (accessed 6/20/17, requires subscription). 